\title{
Etude expérimentale de la couche limite sur fond hydrauliquement lisse soumis à l'action de la houle et/ou du courant.
}

D. Tcheukam-Toko ${ }^{(1)} ;$ F. Loiseau ${ }^{(2)} ; F^{2}$. Marin ${ }^{(3)}$

(1) Doctorant, Laboratoire de Mécanique des Fluides et Génie Civil du Havre (L.M.F.G.C.),Quai frissard - B.P. 265 - 76055 Le Havre Cedex

(2) Doctorant, Laboratoire de Mécanique de Lille - URA 1441- Cité scientifique Bld. P. Langevin - 59655 Villeneuve d'Ascq Cedex

(3) Maître de conférences, Laboratoire de Mécanique des Fluides et Génie Civil du Havre (L.M.F.G.C.), Quai frissard - B.P. 265 - 76055 Le Havre Cedex

Résumé

Pour une recherche de meilleures solutions aux problèmes liés au transport sédimentaire, nous étudions expérimentalement la couche limite sur un fond hydrauliquement lisse soumis à la houle et/ou au courant. Les mesures de vitesse ont été réalisées par Vélocimétrie Doppler Laser à 2 composantes dans le canal de $25 \mathrm{~m}$ du L.M.F.G.C. du Havre. Une étude des distributions de vitesse a été faite le long du canal dans le cas d'un courant seul afin notamment de déterminer la zone où la couche limite est pleinement développée. Nous avons également fait des tests dans le cas de la houle seule avant d'analyser l'effet de la superposition d'une houle au courant sur les distributions de vitesse et les contraintes de frottement pariétal.

\section{Introduction}

Afin d'apporter une contribution à l'étude des problèmes liés au transport sédimentaire tels que l'envasement des ports, la pollution des plages, etc... nous avons effectué une étude expérimentale portant sur la couche limite sur un fond hydrauliquement lisse générée par un courant seul, une houle seule et la superposition de la houle au courant, la houle et le courant se propageant dans le même sens dans cette étude. Si de nombreuses études expérimentales ont déjà été réalisées dans le cas de la houle seule et du courant seul (Jonsson \& Carlsen [4], Sleath [8], Kajiura [5]), il n'en ait pas de même en écoulement combiné houlecourant. A ce sujet, Binder \& Favre-Martinet [2] ont montré que les vitesses près du fond induisaient un retard de phase de la houle quand on superposait le courant à celle-ci. Kemp \& Simons [6] ont montré que la superposition d'une houle à un courant ne modifiait pas la contrainte de frottement sur fond lisse. De nombreuses études numériques ont été également réalisées dans le cas de la houle seule et de la combinaison houle-courant. Huynh-Thanh [3] a mis en évidence le fait que la 
composante horizontale de la contrainte de cisaillement diminue quand on augmente l'angle d'interaction houle-courant. Loiseau \& al. [7] traite également ce problème dans le cas de la houle seule en utilisant une formulation qui prend en compte la surface libre de l'eau. Les tests préliminaires que nous avons effectués dans le cas de la houle seule seront comparés avec ce dernier modèle, avant de faire une analyse de l'effet de superposition du courant à̀ la houle.

\section{Description du dispositif expérimental}

La plate-forme expérimentale est constituée d'un canal à houle récemment aménagé pour pouvoir générer du courant en présence de houle (figure 1).

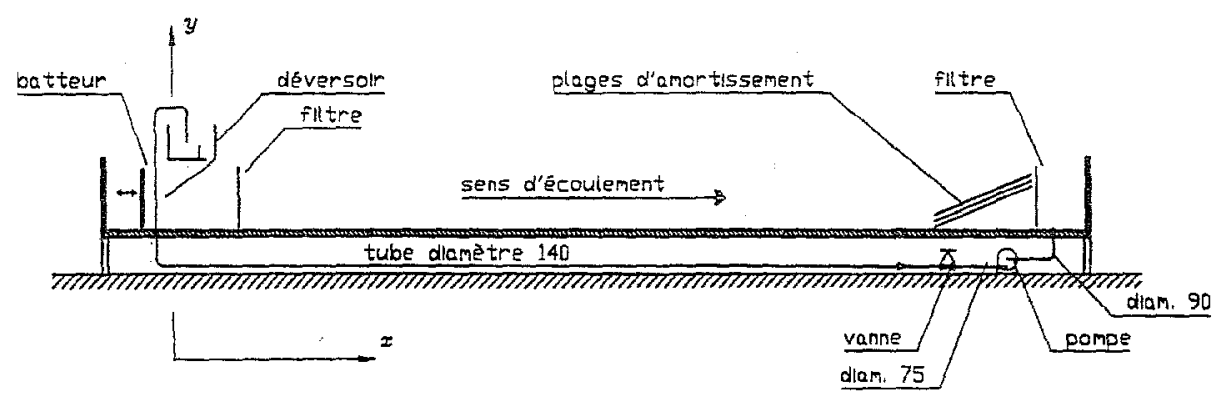

Figure 1: Schéma du canal à houle et/ou à courant.

Le canal à houle a une longueur de $25 \mathrm{~m}$, une largeur de $0,80 \mathrm{~m}$, et une hauteur utile de $1 \mathrm{~m}$. Les parois latérales sont en verre. La houle est générée par un batteur dont le mouvement est piloté par un moto-réducteur à courant asservi par une dynamo tachimétrique. Une plage d'amortissement disposée au bout du canal permet d'obtenir un coefficient de réflexion inférieur à $5 \%$ pour les conditions de houle utilisée [1]. Les études ont été réalisées au-dessus d'un fond lisse en PVC.

Le courant est généré à l'aide d'une pompe centrifuge travaillant en circuit fermé. Le débit peut-être réglé à l'aide d'une vanne située dans la partie refoulement, à $0,3 \mathrm{~m}$ de la pompe (figure 1 ).

Les mesures de vitesses instantanées sont faites par Vélocimétrie Doppler Laser à 2 composantes en diffusion avant. La hauteur d'eau au repos est: $d=0,27 \mathrm{~m}$.

Pour les études réalisées en écoulement périodique, le signal est découpé vague par vague. On construit une vague moyenne, que l'on décompose ensuite en 50 phases. La figure 2 schématise une évolution type de la composante horizontale $U$ de la vitesse au cours d'une période et permet de repérer les différentes phases de l'écoulement, l'origine étant prise au passage du sommet de l'onde au-dessus du point de mesure considéré. 


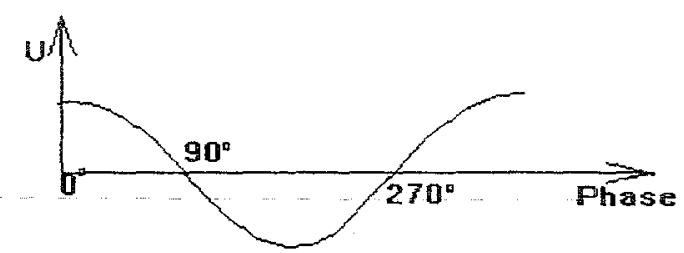

Figure 2: Signal périodique. (Phase: $\phi$ ).

\section{Couche limite générée par le courant seul}

Nous avons réalisé 3 campagnes de mesures caractérisées chacune par une vitesse débitante différente. Pour chaque cas, nous avons obtenu plusieurs profils le long du canal afin de déterminer l'origine de la couche limite dans le canal et la zone où la couche limite est pleinement développée.

\begin{tabular}{|c|c|c|c|c|}
\hline Tests & $\mathrm{V}_{\mathrm{d}}(\mathrm{mm} / \mathrm{s})$ & $\mathrm{u}^{*}(\mathrm{~mm} / \mathrm{s})$ & $\mathrm{y}_{\mathrm{r}}(\mathrm{mm})$ & $\mathrm{R}_{\mathrm{e}}\left(\mathrm{V}_{\mathrm{d}} \mathrm{d} / \mathrm{v}\right)$ \\
\hline $\mathrm{C} 1$ & 15,0 & 0,82 & 12,2 & 4050 \\
$\mathrm{C} 2$ & 30,0 & 1,97 & 5,07 & 8100 \\
$\mathrm{C} 3$ & 60,0 & 3,24 & 3,08 & 16200 \\
\hline
\end{tabular}

Tableau 1: Caractéristique de l'écoulement dans le cas du courant seul $(X=11 \mathrm{~m})$. $R_{e}$ : Nombre de Reynolds.
a) $\mathrm{X}=0,75 \mathrm{~m}$

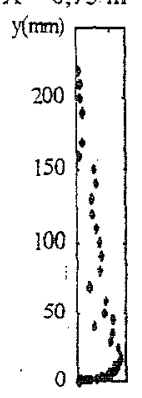

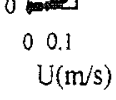
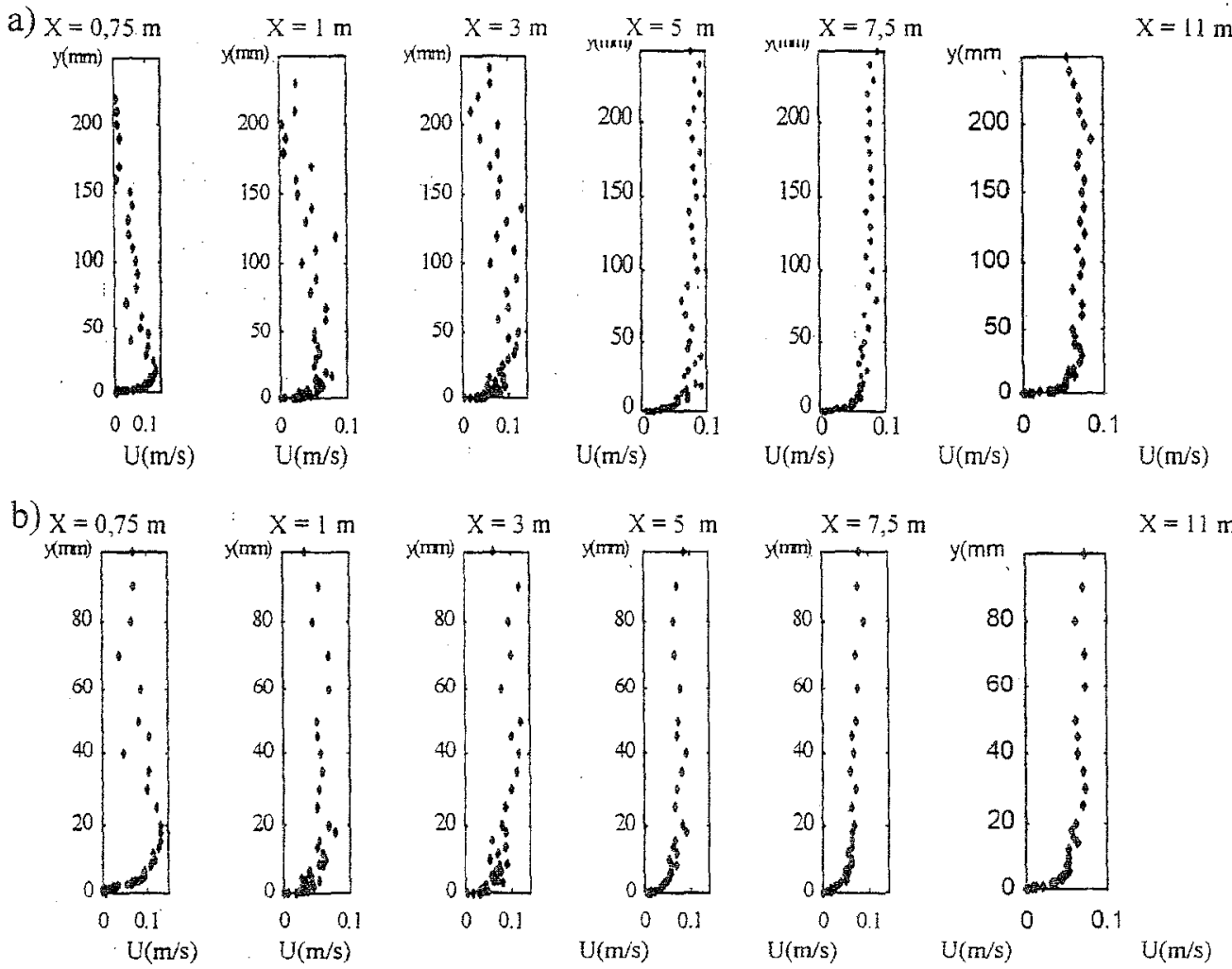

Figures 3: Distribution de vitesse pour $V_{d}=60 \mathrm{~mm} / \mathrm{s}$. a): Toute la hauteur d'eau; b): Près du fond; $X$ : Distance entre le batteur et l'abscisse du profil de vitesse considéré. 
A travers les distributions de vitesse représentées sur les figures $3 \mathrm{a}$ et $3 \mathrm{~b}$ pour une vitesse débitante de $60 \mathrm{~mm} / \mathrm{s}$, on remarque que la couche limite se développe pleinement à partir d'une distance de $5 \mathrm{~m}$ du batteur. Pour les deux autres campagnes faites $\left(V_{d}=30 \mathrm{~mm} / \mathrm{s}\right.$ et $\left.V_{d}=15 \mathrm{~mm} / \mathrm{s}\right)$, on obtient une couche limite pleinement développée respectivement à 7 et $7,5 \mathrm{~m}$ du batteur. Analysons les profils de vitesse obtenus dans la zone où la couche limite est pleinement développée.

La figure 4 représente les profils de vitesse moyenne à $11 \mathrm{~m}$ du batteur; ces profils peuvent être représentés sous forme logarithmique pour obtenir facilement la vitesse de frottement $\mathrm{u}^{*}$ à l'aide des relations:

$$
\begin{aligned}
U^{*} & =A \log (y)+B \\
u^{*} & =A K
\end{aligned}
$$

où $K$ est la contante de $\operatorname{Karman}(K=0,4)$, et $A, B$ les constantes obtenues expérimentalement.

Il est commode d'utiliser les variables de paroi $\mathrm{U}^{+}$et $\mathrm{Y}^{+}$définies par les équations (3) et (4), pour représenter les distributions de vitesse. La figure 5 présente les résultats obtenus pour le test $\mathrm{C} 2$.

$$
\begin{aligned}
& \mathrm{U}^{+}=\mathrm{U} / \mathrm{u}^{*} \\
& \mathrm{Y}^{+}=\left(\mathrm{y} \mathrm{u}^{*} / \mathrm{v}\right)
\end{aligned}
$$

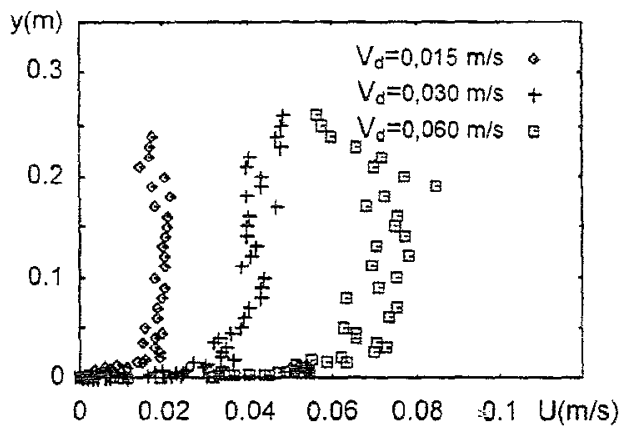

Figure 4: Distributions de vitesse à $\mathrm{X}=1 \mathrm{~lm}$

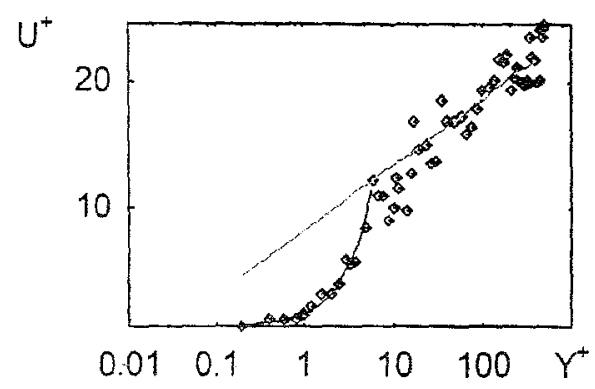

Figures 5: Profil de vitesse en variable de paroi $U^{+}=2,3 \log \left(\mathrm{Y}^{+}\right)+3,6 . \mathrm{V}_{\mathrm{d}}=30 \mathrm{~m} / \mathrm{s}$

Cette figure montre clairement lexistence de deux régions dans la couche limite, l'une loin de la paroi contrôlée par la turbulence, et l'autre près de la paroi dominée par la viscosité. La zone proche de la paroi, la sous-couche de regénerescence qui s'étend jusqu'à $\mathrm{Y}^{+}=10$, a une hauteur donnée par l'équation (5).

$$
\mathrm{y}_{\mathrm{r}}=10 \mathrm{v} / \mathrm{u}^{*}
$$

La région au-dessus, qui s'étend jusqu'à la surface de l'eau, est caractérisée par l'équation (6). La couche limite est donc pour ces trois cas étalée sur toute la hauteur d'eau.

$$
\mathrm{U}^{+}=\mathrm{A}_{1} \log \left(\mathrm{Y}^{+}\right)+\mathrm{B}_{\mathrm{I}}
$$

$A_{1}, B_{1}$, étant des constantes déterminées expérimentalement.

La hauteur de la sous-couche de regénérescence $\mathrm{y}_{\mathrm{r}}$ diminue quand le nombre de Reynolds augmente. 
Les figures $6 a$ et $6 b$ représentent les distributions des écart-type de la vitesse évaluées à partir des vitesses instantanées mesurées $u_{i}$, en utilisant la relation (7):

$$
\sqrt{\mathrm{u}^{2}}=\sqrt{\frac{\sum \mathrm{u}_{\mathrm{i}}^{2} \Delta \mathrm{t}_{\mathrm{i}}}{\sum \Delta \mathrm{t}_{\mathrm{i}}}-U^{2}} \quad \text { avec } \quad U=\frac{\sum u_{i} \Delta t_{i}}{\sum \Delta t_{i}}
$$

$\Delta t_{\mathrm{i}}:$ Période d'échantillonnage.

$\mathrm{U}$ : Vitesse moyenne au point de mesure considéré.

Dès que l'on s'écarte du voisinage immédiat du fond, le niveau de turbulence reste approximativement constant.

a)

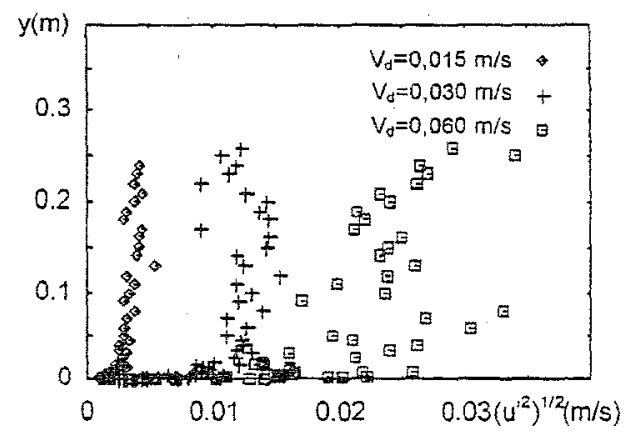

b)

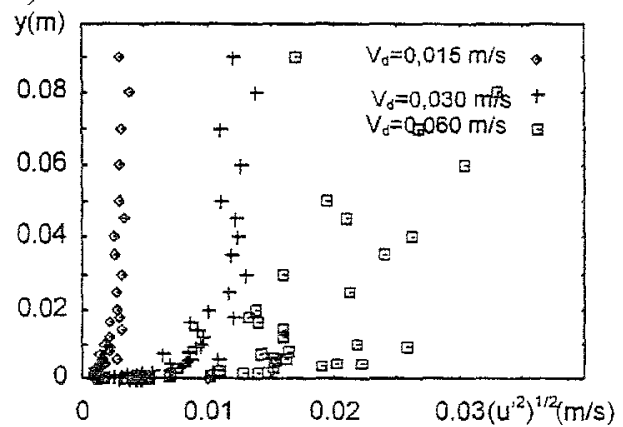

Figures 6: Distributions des écart-type de $U(\mathrm{~m} / \mathrm{s})$.

a) Sur toute la hauteur d'eau; b) Près du fond.

\section{Couche limite générée par la houle seule}

Dans le cas de la houle seule, nous avons réalisé un seul test dont les conditions expérimentales sont les suivantes:
Période
$\mathrm{T}=1,15 \mathrm{~s}$
Hauteur crête-creux $\mathrm{H}=42 \mathrm{~mm}$
Longueur d'onde $\quad \lambda=1,69 \mathrm{~m}$
Nombre de Reynolds $R_{e}=U_{\infty}$ a/v $=893$
Avec $\mathrm{a}=\mathrm{TU}_{\infty} / 2 \pi$ amplitude orbitale de l'écoulement juste à l'extérieur de la couche limite
et $U_{\infty}$ amplitude de la vitesse juste à l'extérieur de la couche limite.

Les profils de vitesse obtenus sont représentés sur la figure 7 pour différentes phases. Loiseau \& Al. [7] ont développé un modèle numérique pour caractériser la couche limite se développant sous l'action de la houle seule, en suivant en lagrangien l'évolution de la surface libre. Pour les mêmes conditions, les résultats du code de calcul sont représentés sur la figure 7.

Les vitesses obtenues expérimentalement ont un profil similaire à celui du modèle numérique, le module de vitesse n'est cependant pas exactement le même. Ceci peut-être dû à l'incertitude (1 à $2 \mathrm{~mm}$ ) que nous rencontrons dans la mesure de la hauteur crête-creux de la houle. Afin de connaître l'évolution temporelle de la vitesse de frottement à la paroi, nous déterminons le gradient de vitesse au fond en considérant la pente de la droite passant par les points de mesures se trouvant entre le fond et le point précédant l'over flow. En effet, nous avons: 


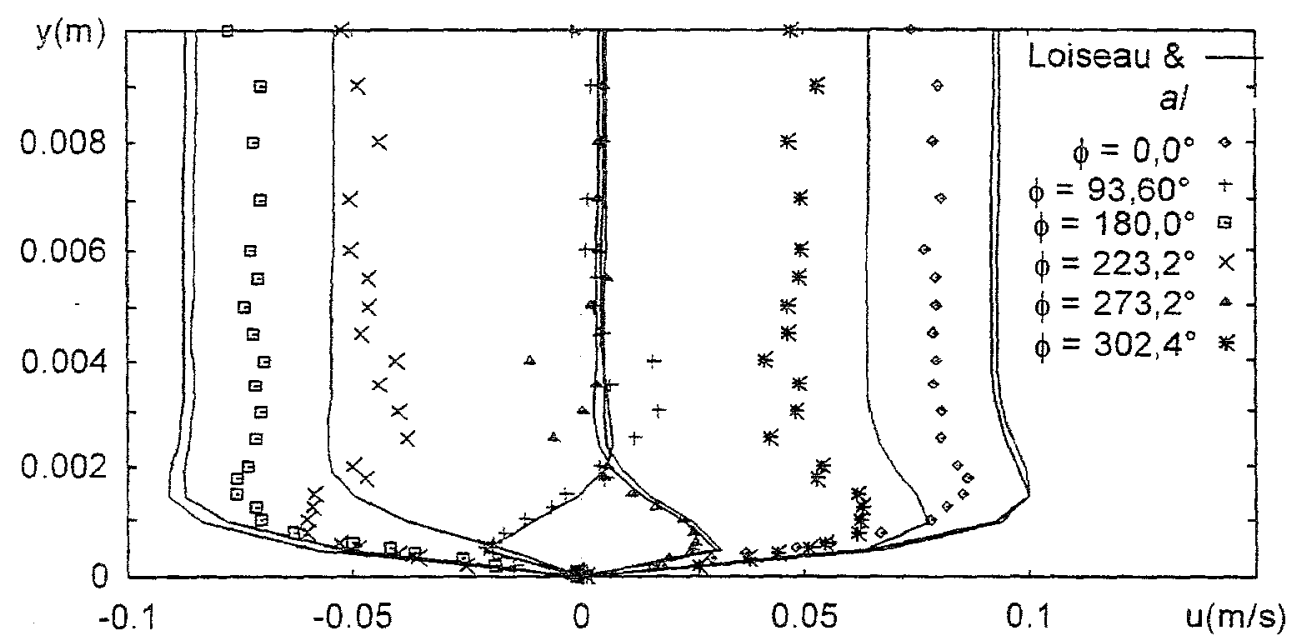

Figure 7: Profils de vitesse moyenne de la houle sur fond lisse.

$$
\begin{aligned}
& \frac{\tau_{0}}{\rho}=v \frac{\partial u}{\partial y} \\
& \left|\mathrm{u}^{*}\right|=\sqrt{\frac{\tau_{0}}{\rho}}
\end{aligned}
$$



Figure 8: Variation temporelle de $\left(\tau_{0} / \rho\right)\left(\mathrm{m}^{2} / \mathrm{s}^{2}\right)$.

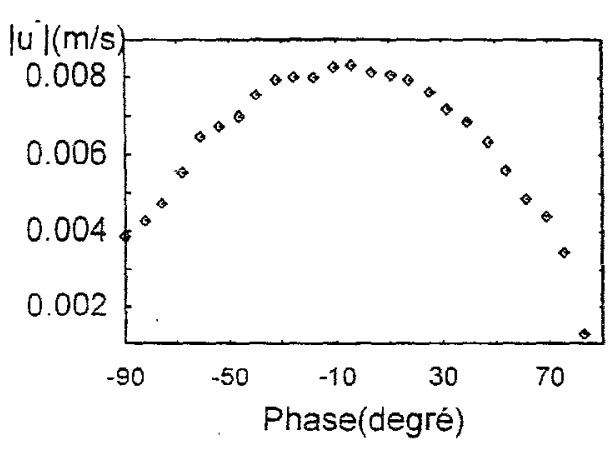

Figure 9 :Variation temporelle de $\left|\mathrm{u}^{*}\right|(\mathrm{m} / \mathrm{s})$ au cours d'une demi période de l'écoulement.

La contrainte de frottement (figure 8) varie entre $-610^{-5}$ et $710^{-5} \mathrm{~m}^{2} / \mathrm{s}^{2}$ lors d'un cycle de la houle, la vitesse de frottement à la paroi (figure 9) ayant un module maximum de $8 \mathrm{~mm} / \mathrm{s}$ au passage du sommet de vague.

\section{Couche limite générée par la combinaison de la houle et du courant}

Nous avons réalisé trois cas d'étude où la houle précédemment décrite, est superposée successivement à chacun des 3 régimes de courant étudiés au paragraphe 3. Les caractéristiques de l'écoulement résultant sont données dans le tableau 2. 


\begin{tabular}{|c|c|c|c|}
\hline Tests & $\mathrm{H}(\mathrm{mm})$ & $\mathrm{V}_{\mathrm{d}}(\mathrm{mm} / \mathrm{s})$ & $\lambda(\mathrm{m})$ \\
\hline $\mathrm{HC} 1$ & 34,0 & 15,0 & 1,8 \\
$\mathrm{HC} 2$ & 23,0 & 30,0 & 1,9 \\
$\mathrm{HC} 3$ & 18,0 & 60,0 & 2,0 \\
\hline
\end{tabular}

Tableau 2: Caractéristiques de l'écoulement combiné houle - courant. $T=1,15 \mathrm{~s}$ pour les trois tests.

Les figures $10 \mathrm{a}, 10 \mathrm{~b}$, et $10 \mathrm{c}$ représentent les profils de vitesse moyenne obtenue respectivement pour les tests $\mathrm{HC} 1, \mathrm{HC} 2$ et $\mathrm{HC} 3$ au creux, au sommet et au front descendant de vague.

L'amplitude de la vitesse près du fond diminue quand on augmente la vitesse débitante dans l'écoulement.

a)

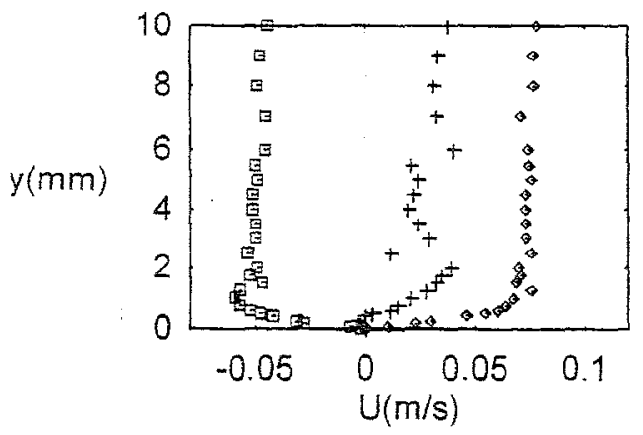

b)

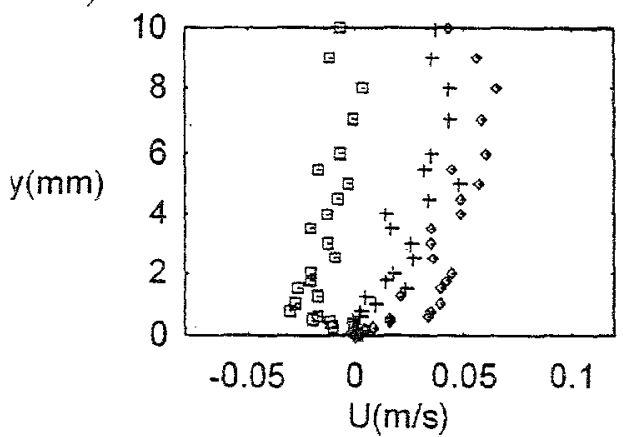

c)

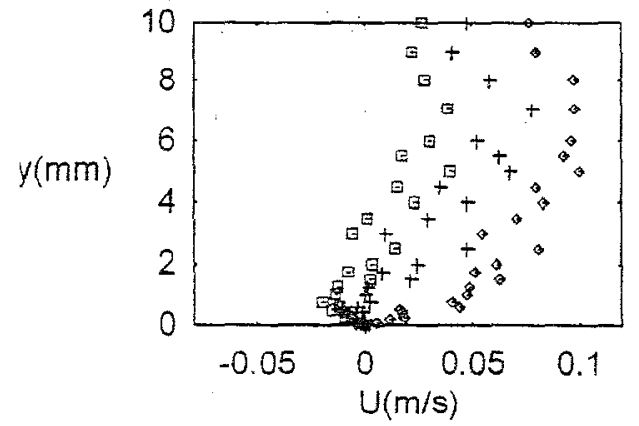

Figures 10: Profils de vitesses moyennes près du fond à $\phi=93,6^{\circ}(+) ; 0^{\circ}(0) ; 180^{\circ}(\square)$ a): $\mathrm{HC} 1$; b): $\mathrm{HC} 2$; c): $\mathrm{HC} 3$.

Le profil logarithmique de la vitesse moyennée sur une période (figure 11) fait apparaitre comme dans le cas du courant seul une couche limite composée de deur régions (sous-couche de regénérescence et région logarithmique). La pente de la droite logarithmique représentée en trait continu permet de retrouver à l'aide de la relation (2), les vitesses moyennes de frottement à la paroi. Selon les critères (10) énoncés par Bijker dans la référence [8], nous pouvons déterminer les tests où soit le courant, soit la houle domine.

$$
\mathrm{U}_{\infty} / \mathrm{u}^{*}>20
$$

Houle dominante

$$
\begin{gathered}
6<U_{\infty} / u^{*}<20 \\
\text { Régime } \\
\text { intermédiaire }
\end{gathered}
$$

$$
\mathrm{U}_{\infty} / \mathrm{u}^{*}<6
$$

Courant dominant 


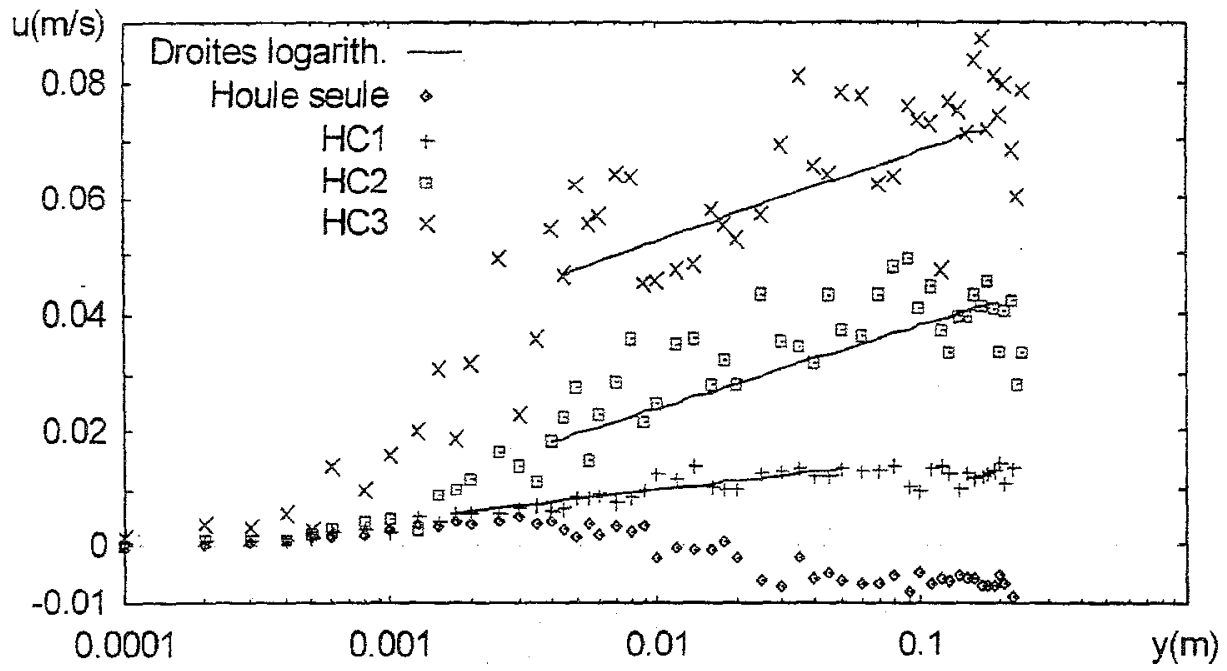

Figure 11: Profil semi-Log de vitesse moyennée sur une période.

\begin{tabular}{|c|c|c|c|c|c|}
\hline Tests & $\mathrm{U}_{\infty}(\mathrm{mm} / \mathrm{s})$ & $\mathrm{u}^{*}(\mathrm{~mm} / \mathrm{s})$ & $\mathrm{U}_{\infty} / \mathrm{u}^{*}$ & $\mathrm{y}_{\mathrm{r}}(\mathrm{mm})$ & \\
\hline $\mathrm{HCl}$ & 60,0 & 0,984 & 60,97 & 20,40 & Houle dominante \\
$\mathrm{HC} 2$ & 40,5 & 2,497 & 16,21 & 8,03 & Régime intermédiaire \\
$\mathrm{HC} 3$ & 19,02 & 3,22 & 5,90 & 6,21 & Courant dominant \\
\hline
\end{tabular}

Tableau 3: Paramètres de l'écoulement combiné houle-courant sur fond lisse.

La présence de la houle dans le courant augmente sensiblement la vitesse de frottement à la paroi par rapport au courant seul, sauf pour le test $\mathrm{HC} 3$ où le courant est dominant. Cette augmentation est environ de $15 \%$ pour $\mathrm{HC1}$, et de $20 \%$ pour $\mathrm{HC} 2$. La comparaison des résultats expérimentaux avec ceux obtenus par addition des vitesses liées à la houle seule et au courant seul (figure 12), nous montre la non-linéarité de l'interaction houle-courant, sauf pour le test $\mathrm{HCl}$ où la houle est dominante.

a)




b)

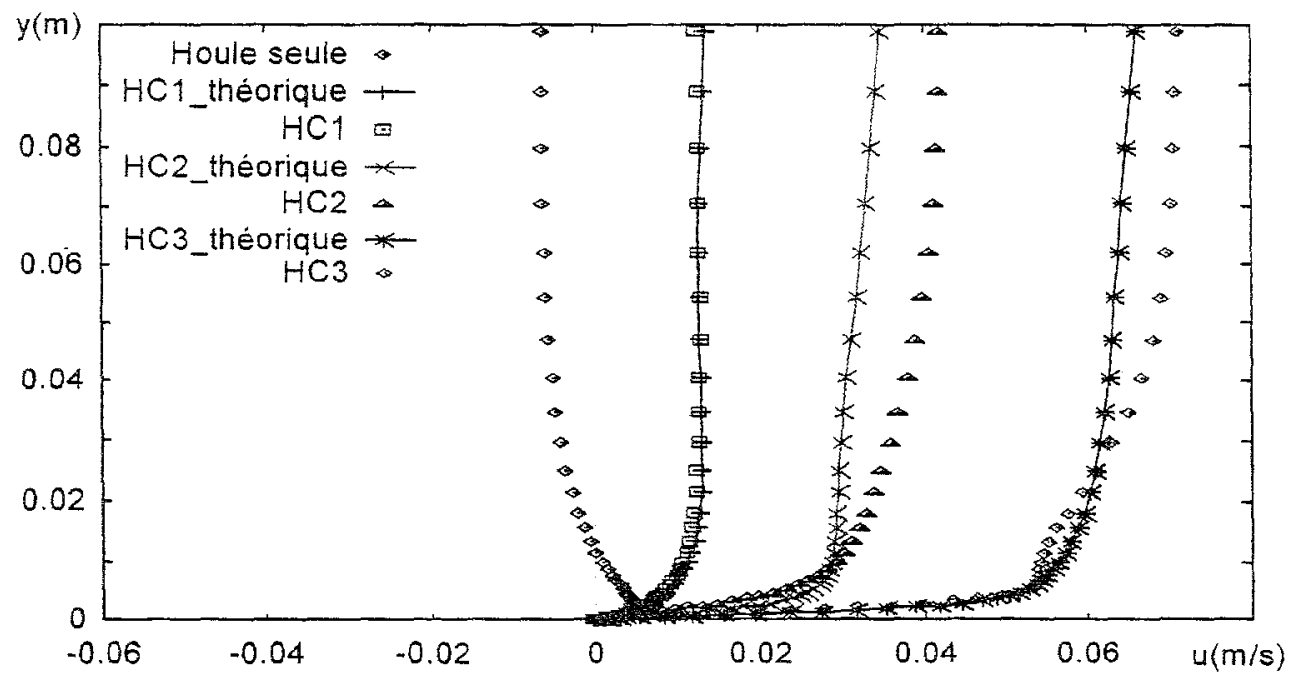

Figure 12: Profils lissés(lissage spline cubique) de vitesse expérimentale et théorique moyennée sur une période. a) Sur toute la hauteur d'eau; b):Près du fond.

Pour les tests $\mathrm{HC} 2$ et $\mathrm{HC} 3$, les vitesses "théoriques"(obtenues par addition), sont plus élevées près du fond et moins élevées dans l'écoulement extérieur par rapport aux vitesses mesurées en écoulement combiné houle-courant. Les tests réalisés par Kemp \& Simons [6] en houle dominante montrent que les vitesses "théoriques" sont plus faibles près du fond et plus élevées loin de la paroi relativement aux résultats expérimentaux. Le régime d'écoulement(houle dominante ou courant dominant), semble donc être un paramètre important dans le mode d'interaction houle-courant. L'épaisseur de la couche limite augmente avec l'addition du courant dans la houle.

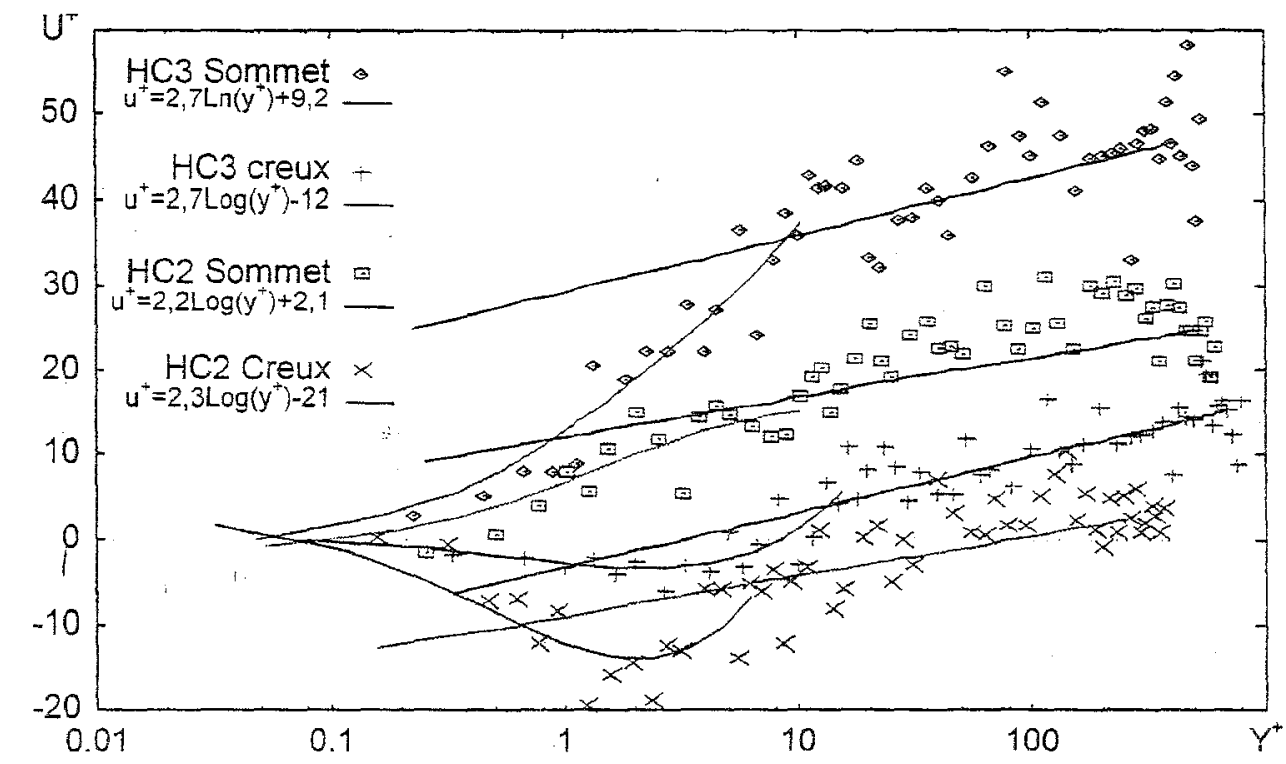

Figure 13:Profil de vitesse en variable de paroi lors du passage de la crête et du creux de vague au-dessus des points de mesure pour les tests $\mathrm{HC} 2$ et $\mathrm{HC} 3$. 
Nous avons évalué la taille moyenne de la sous-couche de regénérescence à l'aide du profil représenté à la figure 13. Elle s'étend jusqu'à environ $\mathrm{Y}^{+}=20$, soit pour les trois tests:

$$
\mathrm{y}_{\mathrm{r}}=20 \mathrm{v} / \mathrm{u}^{*}
$$

\section{Conclusion}

Cette étude nous a permis d'analyser les distributions de vitesse moyenne dans une couche limite générée par un écoulement combiné de houle et de courant audessus d'un fond lisse. Des tests préliminaires en écoulement de courant seul nous ont permis de déterminer la zone dans le canal où la couche limite est pleinement développée. Le profil de vitesse moyenne obéit dans ce cas à une loi logarithmique, caractéristique d'un écoulement turbulent. Le fait de superposer une houle se propageant dans le même sens que le courant augmente la vitesse de frottement à la paroi, ce qui peut faciliter le déplacement des sédiments.

Cette étude sera prolongée par l'analyse de la turbulence dans l'écoulement avant d'étudier l'effet de la superposition de la houle et du courant au-dessus d'un fond rugueux.

\section{Références}

[1] Bélorgey M. \& Le Bas J. Etude par Vélocimétrie Doppler Laser du champs des vitesses instantanées dans une houle produite en laboratoire. La Houille Blanche; No. $5 ;$ pp. 363-368; 1984.

[2] Binder G. \& Favre-Martinet M. The inner layer in unsteady turbulent boundary layers. In Proc. 3rd Meeting of Eurovisc. on Unsteady Turbulent Boundary Layers and Shear Flows; Liverpool; pp. 8-16. 1979.

[3] Huynh-Thanh S. Etude numérique de la couche limite turbulente oscillatoire générée par l'interaction houle-courant en zone côtière. Thèse de doctorat. I.N.P. de Grenoble; 1990.

[4] Jonsson I. G. \& Carlsen N. A. Experimental and theoretical in an oscillatory turbulent boundary layers. J. Hydraul. Res., 14; pp 45-60; 1976.

[5] Kajiura K. A model of the bottom boundary layer in water waves. Bull. Earthquake Res. Inst. 46, pp. 75-123; 1968.

[6] Kemp P. H. et Simons R. R. The interaction between wave and turbulent current: Waves propagating with the current. J. Fluid. Mech. ; Vol. 116; pp 227$250 ; 1982$.

[7] Loiseau F.; Tcheukam-Toko D.; Marin F. Simulation de la couche limite instationnaire générée par un écoulement de houle sur fond lisse. Iv ${ }^{\grave{e}}$ Journées Nationales Génie civil - Génie côtier - Dinard, Avril 1996.

[8] Sleath J.F.A. Sea bed mechanics. Eds. Wiley-Interscience. 1984. 